\title{
Lycorine hydrochloride induces reactive oxygen species-mediated apoptosis via the mitochondrial apoptotic pathway and the JNK signaling pathway in the oral squamous cell carcinoma HSC-3 cell line
}

\author{
MIN-HUI LI ${ }^{1,2^{*}}$, XIN LIAO $^{1,3^{*}}$, CHEN LI $^{3}$, TIAN-TIAN WANG ${ }^{3}$, YI-SONG SUN ${ }^{3}$, KANG YANG $^{2}$, \\ PEI-WEN JIANG ${ }^{2}$, SONG-TING SHI ${ }^{4}$, WEN-XIN ZHANG ${ }^{2}$, KUN ZHANG $^{3}, \mathrm{CHAO} \mathrm{LI}^{5}$ and PING YANG ${ }^{2}$ \\ ${ }^{1}$ Center of Science and Research, ${ }^{2}$ School of Basic Medicine, ${ }^{3}$ School of Bioscience and Technology, \\ and ${ }^{4}$ School of Laboratory Medicine, Chengdu Medical College, Chengdu, Sichuan 610500; \\ ${ }^{5}$ Department of Head and Neck Surgery, Sichuan Cancer Hospital and Institute, Chengdu, Sichuan 610041, P.R. China
}

Received June 23, 2020; Accepted November 18, 2020

DOI: $10.3892 / \mathrm{ol} .2021 .12497$

\begin{abstract}
Poor drug efficacy is a prominent cause of oral squamous cell carcinoma (OSCC) treatment failure. Although increased efforts in developing OSCC therapeutic strategies have been achieved in recent decades, the 5-year survival rate of patients with OSCC remains poor and effective drugs to treat OSCC are lacking. The aim of the present study was to investigate the apoptotic effect caused by lycorine hydrochloride (LH) and to identify its mechanism in the OSCC HSC-3 cell line. The findings demonstrated that LH effectively induced HSC-3 cell apoptosis and cell cycle arrest at the $\mathrm{G}_{0} / \mathrm{G}_{1}$ phase, resulting in the inhibition of cell proliferation. Furthermore, it was found that $\mathrm{LH}$ increased reactive oxygen species (ROS) production, triggered mitochondrial membrane potential (MMP) disorder, enhanced the protein expression levels of Bax, Bim, cleaved caspase-9, caspase- 3 and poly(ADP-ribose) polymerase 1 and decreased Mcl-1 expression. The protein expression levels of important members of the JNK signaling pathway, including phosphorylated (p)-JNK, p-mitogen-activated protein kinase kinase 4 and p-c-Jun, were
\end{abstract}

Correspondence to: Professor Ping Yang, School of Basic Medicine, Chengdu Medical College, 783 Xindu Avenue, Chengdu, Sichuan 610500, P.R. China

E-mail: 920192655@qq.com

Professor Chao Li, Department of Head and Neck Surgery, Sichuan Cancer Hospital and Institute, 55 Section 4 Renmin South Road, Chengdu, Sichuan 610041, P.R. China

E-mail: 1li_chao3@163.com

*Contributed equally

Key words: lycorine hydrochloride, oral squamous cell carcinoma, apoptosis, reactive oxygen species, mitochondrial apoptotic pathway, JNK signaling pathway significantly increased in LH-treated cells, accompanied by an increase in ROS. However, N-acetyl cysteine (NAC), a potent antioxidant, reversed the upregulated mRNA expression of c-Jun, as well as the enhanced ROS production, the disorder of MMP and the apoptosis of HSC-3 cells induced by LH. These results suggested that $\mathrm{LH}$ may induce HSC-3 cell apoptosis via the ROS-mediated mitochondrial apoptotic pathway and the JNK signaling pathway, which indicated that LH may be a potential drug candidate for anti-OSCC therapy.

\section{Introduction}

Oral cancer is a malignant tumor type with an increasing prevalence worldwide, with $\sim 350,000$ new cases $(2.0 \%$ of the total cancer cases) and 170,000 mortalities (1.9\% of the total cancer mortality) reported in 2018 (1-3). Furthermore, the 5-year overall survival rate of patients with advanced stage oral cancer is $30-40 \%$ (1-3). Oral squamous cell carcinoma (OSCC), which has high morbidity and mortality rates, is the most common oral cancer type, accounting for $>90 \%$ of the histological classification in males $(4,5)$. While progress in OSCC diagnosis and therapies has been achieved in recent decades, the primary treatment option for patients with advanced stage OSCC is the combined therapeutic regimen of surgery followed by adjuvant radiotherapy with or without chemotherapy $(6,7)$, and the 5-year survival rate of patients with OSCC remains poor, despite patients responding to the combination of chemotherapy with docetaxel, cisplatin and 5-fluorouracil $(8,9)$.

The leading causes of OSCC treatment failure include metastatic spread, recurrence and poor drug efficacy (10). Furthermore, previous studies have reported that OSCC treatment failure is associated with drug resistance, which is mainly attributed to chemotherapeutic drug transportation, metabolic reprogramming, redox status and DNA repair (11). The absence of effective drugs to treat patients with OSCC and the resistance of existing drugs indicates the urgent requirement for novel and effective anti-OSCC drugs for clinical treatment. 
It has been revealed that the proliferative ability of OSCC cells is associated with oxidative stress $(12,13)$. Increments in reactive oxygen species (ROS) may induce OSCC cell death, and a decreasing trend in ROS is observed in patients with advanced stage OSCC, suggesting that ROS may be an important potential antitumor target in OSCC therapeutic strategies. In recent years, a number of medicinal herbs and their active ingredients have been reported to induce ROS-mediated apoptosis in OSCC cells, including curcumin (10), $\beta$-lapachone (14) and erufosine (15).

Lycorine is the major active ingredient of the Amaryllidaceae alkaloids derived from the medicinal herb Lycoris radiate. Lycorine and its derivatives have previously been reported to possess various biological activities, including antiviral, anti-inflammatory and antitumor activities. Furthermore, lycorine may act on ovarian cancer cells (16), breast cancer cells (17), hepatoma cells (18), melanoma cells (19) and bladder cancer cells (20) to induce apoptosis and proliferation, and inhibit tumor neovascularization. However, to the best of our knowledge, the anti-OSCC effects of lycorine have not previously been reported.

The present study aimed to investigate the apoptosis of the OSCC HSC-3 cell line induced by lycorine hydrochloride (LH), and to investigate the changes of ROS and the expression levels of the apoptosis-related proteins, including Bax, Bim, Caspase, poly(ADP-ribose) polymerase 1 (PARP), JNK, mitogen-activated protein kinase kinase 4 (MKK4) and c-JUN, in order to identify the apoptotic pathways.

\section{Materials and methods}

Materials. LH (purity $\geq 98 \%$ ) was purchased from Man Si-Te (http://www.cdmust.com/). Dulbecco's modified Eagle's medium (DMEM) and fetal bovine serum (FBS) were purchased from Gibco; Thermo Fisher Scientific, Inc. A Cell Counting kit (CCK)-8 was purchased from Dojindo Molecular Technologies, Inc. N-acetyl-L-cysteine (NAC; cat. no. A7250; purity $\geq 99 \%$ ) and propidium iodide (PI) were obtained from Sigma-Aldrich (Merck KGaA). PhosSTOP phosphatase inhibitor cocktail, complete $^{\mathrm{TM}}$ protease inhibitor cocktail, Annexin V-FITC/PI apoptosis detection kit, JC-1 detection kit and TaqMan probe were obtained from Roche Diagnostics, Inc. Horseradish peroxidase (HRP) chemiluminescence kit and polyvinylidene difluoride (PVDF) membranes were obtained from EMD Millipore. An intracellular ROS detection kit and RIPA buffer were obtained from BI Yun-Tian (https://www.beyotime. com/index.htm).

The antibodies against GAPDH (cat. no.5174; 1:1,000), Bax (cat. no. 5023; 1:1,000), Bim (cat. no. 2933; 1:1,000), Bid (cat. no. 2002; 1:1,000), Mcl-1 (cat. no. 5453; 1:1,000), caspase9 (cat. no. 9502; 1:1,000), caspase3 (cat. no. 14220; 1:1,000), PARP (cat. no. 9542; 1:1,000), JNK (cat. no. 9252; 1:1,000), c-Jun (cat. no.9165; 1:1,000), MKK4 (cat. no. 9152; 1:2,000) and ATF2 (cat. no. 35031; 1:2,000), as well as the phospho-JNK pathway antibody sampler kit (cat. no. 4668; 1:1,000) and goat anti-rabbit IgG antibody (cat. no. 7074;1:2,000) were purchased from Cell Signaling Technology, Inc.

RNAiso Plus and PrimeScript ${ }^{\mathrm{TM}}$ RT Reagent kit were purchased from Takara Bio, Inc. The synthetic primers were synthesized by Qingke Biological Company (http://www. tsingke.net). All other chemicals were of analytical grade.

Cell culture. The human OSCC HSC-3, HSC-4, UM1 and UM2 cell lines were donated by Professor Wu Mingbo's research group at the State Key Laboratory of Biotherapy of Sichuan University (Chengdu, Sichuan, China). The cells were cultured in DMEM supplemented with $10 \%$ FBS, and $100 \mathrm{U} / \mathrm{ml}$ penicillin and streptomycin, under humidified conditions with $5 \% \mathrm{CO}_{2}$ at $37^{\circ} \mathrm{C}$, according to the culture conditions recommended by the American Type Culture Collection. Cells in the logarithmic growth phase, which was when the cells reached a high density of $\sim 80 \%$, were used in the subsequent experiments.

Cell proliferation assay. The cell viability was detected using a CCK-8 assay. Viable HSC-3 cells were seeded onto a 96-well plate and incubated with appropriate concentrations of $\mathrm{LH}$, followed by the addition of CCK-8 into each well for another $4 \mathrm{~h}$ at $37^{\circ} \mathrm{C}$ with $5 \% \mathrm{CO}_{2}$. The absorbance at $450 \mathrm{~nm}$ was measured using a spectra microplate spectrophotometer (BioTek Powerwave; BioTek Instruments, Inc.). Data acquisition and the $\mathrm{IC}_{50}$ analysis were performed using GraphPad Prism 7 software (GraphPad Software, Inc.).

Cell cycle assay. HSC-3 cells treated with LH for 12 or $24 \mathrm{~h}$ were harvested and washed briefly with ice-cold PBS. Cells were fixed in $75 \%$ ice-cold ethanol at $4^{\circ} \mathrm{C}$ overnight and concentrated following the removal of ethanol. The cellular DNA was stained with PI at $4^{\circ} \mathrm{C}$ for $30 \mathrm{~min}$ in dark. Data acquisition and analysis of the cell cycle distribution were performed using a flow cytometer (BD FACS Accuri C6; BD Biosciences) and CFlow Sampler v. 1.0 software (BD Biosciences).

Cell apoptosis assay. Viable HSC-3 cells treated with different concentrations of LH (with or without pre-treatment of $5 \mathrm{mM}$ $\mathrm{NAC}$ for $1 \mathrm{~h}$ ) for $24 \mathrm{~h}$ were harvested and washed with ice-cold PBS. Cells were dual-stained with Annexin V-FITC and PI at $25^{\circ} \mathrm{C}$ for $20 \mathrm{~min}$, followed by FCM analysis according to the manufacturer's protocol of the Annexin V-FITC/PI apoptosis detection kit. Data acquisition and analysis of the cell apoptosis were performed using a flow cytometer (BD FACS Accuri C6; BD Biosciences) and CFlow Sampler v.1.0 software (BD Biosciences).

Intracellular ROS assay. To monitor the generation of intracellular ROS, viable HSC-3 cells were pre-treated with NAC for $1 \mathrm{~h}$ at $37^{\circ} \mathrm{C}$, followed by 10 and $20 \mu \mathrm{M}$ of $\mathrm{LH}$ for $24 \mathrm{~h}$. The generation of intracellular ROS was detected via flow cytometry with 2',7'-dichlorodihydrofluorescein diacetate (DCFH-DA) as the peroxide-sensitive fluorescent probe, which may be converted to DCFH and then oxidized to the fluorescent compound DCF in the presence of ROS in cells. The cells treated with NAC and LH were harvested, washed with PBS, mixed with $10 \mu \mathrm{M}$ DCFH-DA and incubated at $25^{\circ} \mathrm{C}$ for $30 \mathrm{~min}$ in the dark. The cell suspension was subjected to the flow cytometer and the fluorescence signal was detected for intracellular ROS measurement via flow cytometry (BD FACS Accuri C6; BD Biosciences) and data analysis of intracellular ROS was performed using a CFlow Sampler v.1.0 software (BD Biosciences). 
A
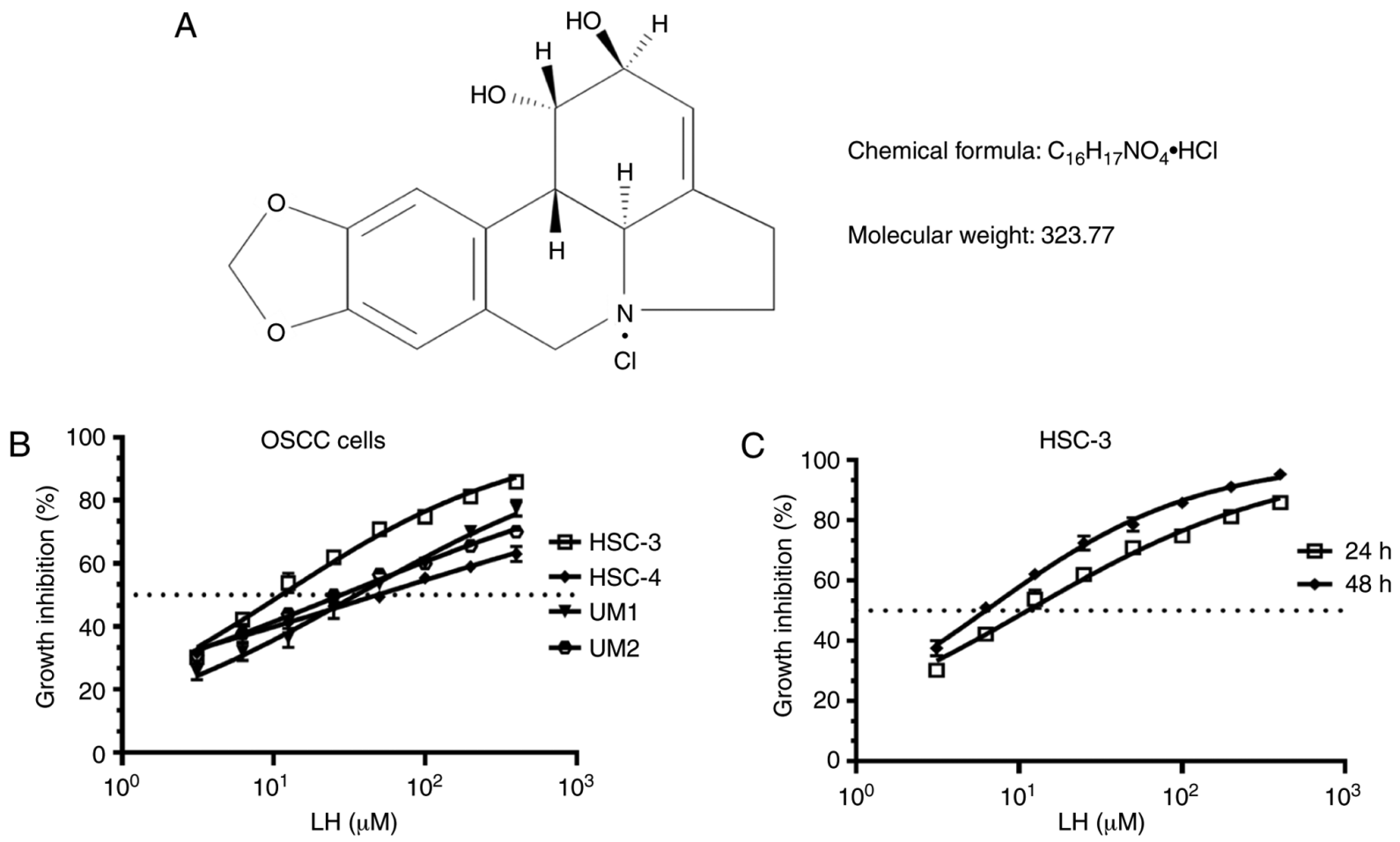

Figure 1. LH inhibits OSCC cell proliferation in vitro. (A) Chemical structure of LH. (B) HSC-3, HSC-4, UM1 and UM2 OSCC cell lines were incubated with $\mathrm{LH}$ at the indicated concentrations for $24 \mathrm{~h}$, and cell proliferation was measured using a CCK-8 assay. (C) HSC-3 cells were incubated with LH at the indicated concentrations for 24 and $48 \mathrm{~h}$, and cell proliferation was measured using a CCK-8 assay. IC50 value of LH-treated OSCC cells was calculated with GraphPad Prism 7 software. OSCC, oral squamous cell carcinoma; LH, lycorine hydrochloride; CCK-8, Cell Counting kit-8.

Mitochondrial membrane potential (MMP) assay. The changes in MMP were detected via staining cells with JC-1, a fluorescent probe for MMP detection. HSC-3 cells treated with $\mathrm{LH}$ (with or without pre-treatment of $5 \mathrm{mM}$ NAC for $1 \mathrm{~h})$ for $24 \mathrm{~h}$ were harvested, washed with ice-cold PBS and stained with $5 \mathrm{mg} / \mathrm{ml} \mathrm{JC}-1$ at $37^{\circ} \mathrm{C}$ for $30 \mathrm{~min}$ in the dark. Data acquisition and analysis of MMP were performed by flow cytometry (BD FACS Accuri C6; BD Biosciences) and CFlow Sampler v.1.0 software (BD Biosciences).

Western blot analysis. HSC-3 cells treated with LH for $24 \mathrm{~h}$ were lysed in RIPA buffer with protease inhibitor or phosphatase inhibitor, and the lysates were centrifuged at $13,000 \mathrm{x}$ g for $15 \mathrm{~min}$ at $4^{\circ} \mathrm{C}$. The protein concentration was determined using the BCA method. Equal amounts (50 $\mu \mathrm{g} /$ lane) of total proteins were separated via $12 \%$ SDS-PAGE and the proteins were transferred onto PVDF membranes via wet electro-transfer for $150 \mathrm{~min}$ at $250 \mathrm{~mA}$. The membranes were blocked for $1.5 \mathrm{~h}$ with $5 \%$ skimmed milk at room temperature and incubated overnight at $4^{\circ} \mathrm{C}$ with the aforementioned primary antibodies, followed by incubation with the HRP-conjugated secondary antibody for $1.5 \mathrm{~h}$ at room temperature. The blots were visualized using the enhanced chemiluminescence system and densitometry was performed using Quantity One v.4.6 analysis software (Bio-Rad ChemiDocXRS; Bio-Rad Laboratories, Inc.).

Reverse transcription-quantitative polymerase chain reaction $(R T-q P C R)$ analysis. HSC-3 cells treated with LH (with or without pre-treatment of $5 \mathrm{mM}$ NAC for $1 \mathrm{~h}$ ) for $24 \mathrm{~h}$ were harvested. Total RNA was extracted from the cultured cells using RNAiso Plus, and cDNAs were synthesized using the PrimeScript RT reagent kit. The conditions for RT were as follows: $42^{\circ} \mathrm{C}$ for $2 \mathrm{~min}, 37^{\circ} \mathrm{C}$ for $15 \mathrm{~min}, 85^{\circ} \mathrm{C}$ for $5 \sec 4^{\circ} \mathrm{C}$ for $30 \mathrm{~min}$. qPCR was performed with the Bio-Rad CFX96 Real-Time PCR Detection system using the TaqMan probe method. The following primers were used: c-Jun forward, 5'-CCAAAGGATAGTGCGATGTTT-3' and reverse, 5'-CTG TCCCTCTCCACTGCAAC-3'; and GAPDH forward, 5'-AGC CACATCGCTCAGACAC-3' and reverse, 5'-AATACGACC AAATCCGTTGACT-3'. The thermocycling conditions were as follows: $95^{\circ} \mathrm{C}$ for $10 \mathrm{~min}$, followed by 45 cycles at $95^{\circ} \mathrm{C}$ for $10 \mathrm{sec}, 60^{\circ} \mathrm{C}$ for $30 \mathrm{sec}$, and at $40^{\circ} \mathrm{C}$ for $30 \mathrm{sec}$. The mRNA expression levels were calculated with the $2^{-\Delta \Delta \mathrm{Cq}}$ method (21) and expressed in relative quantification units. A control without cDNA was run in parallel with each assay. Each reaction was performed in triplicate.

Statistical analysis. All experiments were repeated $\geq 3$ times, unless otherwise stated. Data are presented as the mean \pm standard deviation and were compared using a Dunnett's test for multiple group comparisons with the control and Tukey's test for comparisons of differences between group using GraphPad Prism 7 software (GraphPad Software, Inc.). $\mathrm{P}<0.05$ was considered to indicate a statistically significant difference.

\section{Results}

LH inhibits the proliferation of OSCC cells. Several OSCC cell lines, including HSC-3, HSC-4, UM1 and UM2, were originally used to investigate the suppressive effect on proliferation induced by LH in vitro (Fig. 1A), based on the 
previously reported antitumor effects of $\mathrm{LH}$ in other malignant tumors (16-20). The results demonstrated that LH inhibited the proliferation of HSC-3, HSC-4, UM1 and UM2 cells in vitro, with $\mathrm{IC}_{50}$ values of $15.65,48.55,35.32$ and $27.95 \mu \mathrm{M}$, respectively (Fig. 1B). Comparison of the growth inhibitory activity of LH on these four OSCC cell lines indicated that the HSC-3 cell line had the highest sensitivity to LH. LH inhibited HSC-3 cell proliferation in a time- and dose-dependent manner, with a $24 \mathrm{~h} \mathrm{IC}_{50}$ value of $15.65 \mu \mathrm{M}$ and a $48 \mathrm{~h} \mathrm{IC}_{50}$ value of $6.23 \mu \mathrm{M}$ (Fig. 1C). Taken together, these data suggested that LH may inhibit the proliferation of OSCC cells in vitro, particularly that of HSC-3 cells. Therefore, HSC-3 cells were used in the subsequent studies.

LH induces HSC-3 cell cycle arrest at the $G_{0} / G_{1}$ phase. The effect of LH on cell cycle progression in HSC-3 cells was investigated to elucidate the mechanism via which $\mathrm{LH}$ exerts its antiproliferative activity. HSC-3 cells were treated with LH for $12 \mathrm{~h}$, followed by the staining of the cellular DNA with PI and flow cytometric analysis. The cell cycle assay demonstrated that the proportion of HSC-3 cells in the $\mathrm{S}$ and $\mathrm{G}_{2} / \mathrm{M}$ phases decreased from 16.2 and $25.5 \%$ (without $\mathrm{LH}$ ) to 12.5 and $20.1 \%$ (in the presence of $20 \mu \mathrm{M} \mathrm{LH}$ ), respectively (Fig. 2A and B). By contrast, the percentage of cells in the $\mathrm{G}_{0} / \mathrm{G}_{1}$ phase increased from $54.0 \%$ (without $\mathrm{LH}$ ) to $60.5 \%$ (in the presence of $20 \mu \mathrm{M} \mathrm{LH}$ ), indicating an $\mathrm{LH}$-induced cell cycle arrest at the $G_{0} / G_{1}$ transition in HSC-3 cell cycle progression (Fig. 2A and C). Furthermore, the proportion of HSC-3 cells in the sub- $\mathrm{G}_{0} / \mathrm{G}_{1}$ phase increased by $\sim 12$-fold in a dose-dependent manner from $1.3 \%$ (without $\mathrm{LH}$ ) to $15 \%$ (in the presence of $40 \mu \mathrm{M} \mathrm{LH}$ ) (Fig. 2A and B). Following HSC-3 cells being treated with $\mathrm{LH}$ for $24 \mathrm{~h}$, the proportion of HSC-3 cells in sub- $\mathrm{G}_{0} / \mathrm{G}_{1}$ phases increased significantly in a dose-dependent manner from $2.1 \%$ (without $\mathrm{LH}$ ) to $24.8 \%$ (in the presence of $40 \mu \mathrm{M} \mathrm{LH}$; Fig. 2D-F), indicating that apoptosis may be induced by LH in HSC-3 cells. Therefore, these data suggested that $\mathrm{LH}$ induced HSC-3 cell cycle arrest at the $\mathrm{G}_{0} / \mathrm{G}_{1}$ phase, and that cell apoptosis may be the inhibitory mechanism in HSC-3 cell proliferation induced by LH.

LH induces apoptosis mediated by ROS in HSC-3 cells. To further assess the apoptotic effect of LH, HSC-3 cells were identified via dual-staining with Annexin V-FITC and PI, followed by flow cytometric analysis. A dose-dependent increase in the percentage of apoptotic cells was observed in the presence of 0,10 and $20 \mu \mathrm{M}$ LH with apoptotic rates of $7.4,24.9$ and $35.6 \%$, respectively. Furthermore, the early apoptosis and late apoptosis of HSC-3 cells increased from 3.0 and $4.4 \%$ (without $\mathrm{LH}$ ) to 18.4 and $17.2 \%$ (in the presence of $20 \mu \mathrm{M}$ $\mathrm{LH})$, respectively. It was also identified that NAC reversed the apoptotic rate from 35.6 to $23.8 \%$ (Fig. $3 \mathrm{~A}$ and $\mathrm{C}$ ).

To investigate whether intracellular ROS, which are well-known signaling molecules serving a pivotal role in mediating cell apoptosis, were associated with LH-induced apoptosis in HSC-3 cells, the intracellular ROS levels were investigated using DCFH-DA. The results indicated that LH significantly increased ROS levels in HSC-3 cells from $2.8 \%$ (without $\mathrm{LH}$ ) to $35.4 \%$ (in the presence of $20 \mu \mathrm{M}$ LH), which was a $\sim 13$-fold increase (Fig. 3B and D). By contrast, pre-treatment with NAC, a ROS inhibitor, reversed the ROS levels from 35.4 to $13.1 \%$, suggesting that $\mathrm{LH}$ had a ROS-inducing effect (Fig. 3B and D). Therefore, it was indicated that LH may induce apoptosis mediated by ROS in HSC-3 cells.

LH induces HSC-3 cell apoptosis via a mitochondrial pathway. The mitochondrial apoptotic pathway is a well-known, important pathway in programmed cell death (22). To investigate whether the mitochondrial pathway was involved in LH-induced HSC-3 cell apoptosis, the change of MMP $(\Delta \Psi \mathrm{m})$, an important factor for mitochondrial dysfunction, was measured with JC-1 staining via flow cytometry. In mitochondria, the dissipated MMP may prevent the accumulation of JC-1, leading to a shift from red (JC-1 aggregates) to green fluorescence (JC-1 monomers). These results demonstrated that LH depolarized the MMP in a dose-dependent manner in the presence of 0,10 and $20 \mu \mathrm{M} L H$ with a $\Delta \Psi \mathrm{m}$ of $5.7,17.8$ and $32.2 \%$, respectively. Furthermore, the depolarized MMP could be recovered from $32.2 \%$ (in the presence of $20 \mu \mathrm{M} \mathrm{LH}$ ) to $18.3 \%$ when the HSC-3 cells were pre-treated with NAC (Fig. 4A and B).

Caspase-9 is a key mediator in the intrinsic mitochondrial-mediated apoptotic pathway, which may subsequently activate caspase-3 and poly ADP-ribose polymerase (PARP), leading to degradation of cellular components for apoptosis. Based on the significant roles of these caspases involved in apoptosis, the catalytic activities of these caspases were measured by western blot analysis. A prominent increase in the levels of cleaved caspases-9, caspase-3 and PARP were identified in an LH dose-dependent manner. Furthermore, expression level changes of several critical members of the Bcl-2 family targeting the mitochondrial apoptotic pathway, including Bax, Bim and Mcl-1, were analyzed. The western blot analysis results identified that LH upregulated the expression levels of the pro-apoptotic members, Bax and Bim, but downregulated the expression of the anti-apoptotic protein, Mcl-1, in a dose-dependent manner, while it did not significantly affect the expression of the pro-apoptotic member, Bid (Fig. 4C-E). These results indicated that the mitochondrial pathway was involved in ROS-mediated apoptosis induced by LH.

JNK signaling pathway is involved in LH-induced apoptosis in HSC-3 cells. A previous study reported that the JNK signaling pathway modulates mitochondrial pathway-induced cell apoptosis triggered by oxidative stress, leading to mitochondrial dysfunction and cell death (23). The results of the present study demonstrated that the mRNA expression levels of transcription factor c-Jun were increased 35-fold compared with the control following HSC-3 cells being treated with $20 \mu \mathrm{M}$ LH. Furthermore, this increase was significantly decreased when the HSC-3 cells were pre-treated with NAC (Fig. 5A). This result indicated that LH induced the mRNA expression of c-Jun via ROS in HSC-3 cells. The protein expression levels of p-MKK4 and p-JNK, as well as the phosphorylated transcription factors c-Jun and ATF2, were detected to investigate whether the JNK signaling pathway serves vital roles in apoptosis when HSC-3 cells were exposed to LH. Upregulated expression levels of p-MKK4 and p-JNK were identified in LH-treated HSC-3 cells. Additionally, 


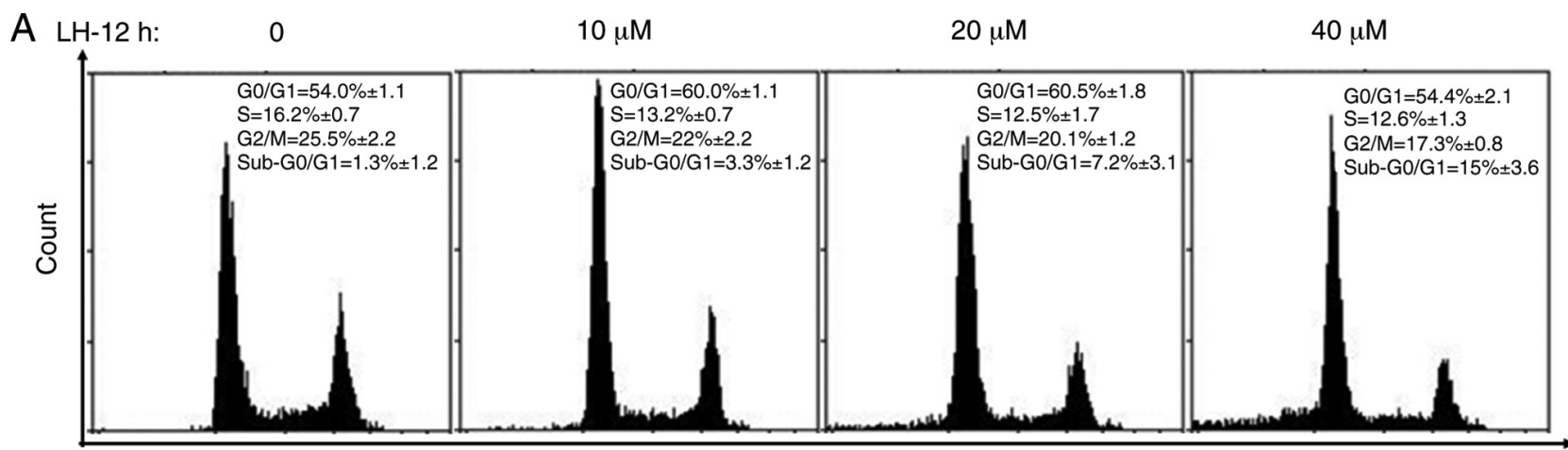

$\mathrm{Pl}$
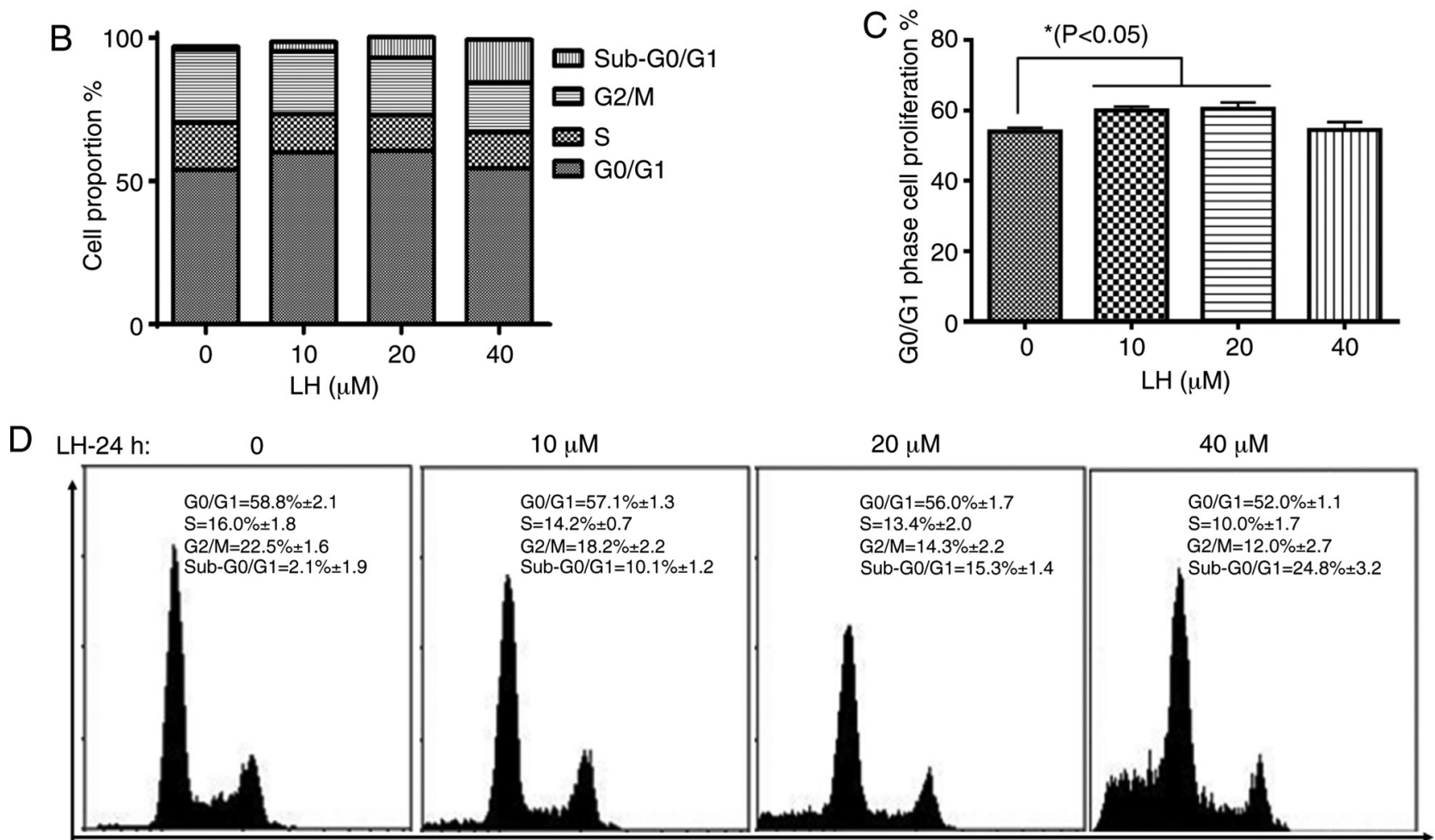

$\mathrm{PI}$
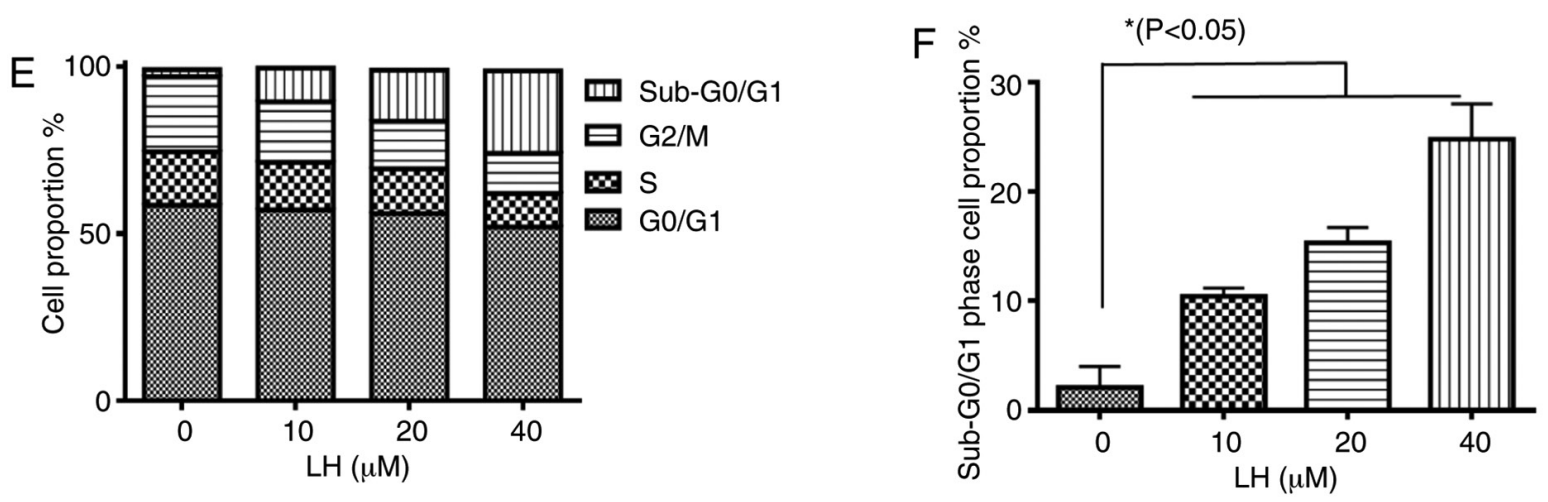

Figure 2. Inhibitory effect of LH on HSC-3 cell cycle. (A) Following treatment of HSC-3 cells with LH at the indicated concentrations for $12 \mathrm{~h}$, the DNA content of propidium iodide-stained cells was detected using FCM; (B) total cell proportions and (C) the $\mathrm{G}_{0} / \mathrm{G}_{1}$ phase cell proportion were analyzed by FCM and GraphPad Prism 7 software, respectively. (D) Following treatment of HSC-3 cells with LH at the indicated concentrations for $24 \mathrm{~h}$, the DNA content of propidium iodide-stained cells was detected using FCM; (E) total cell proportions and (F) the $G_{0} / G_{1}$ phase cell proportion were analyzed by FCM and GraphPad Prism 7 software, respectively. ${ }^{*} \mathrm{P}<0.05$ was considered to indicate a statistically significant different using Dunnett's test for multiple group comparisons with the control group $(0 \mu \mathrm{M} \mathrm{LH})$. LH, lycorine hydrochloride.

the upregulated expression level of the transcription factor p-c-Jun was observed. However, LH did not notably affect
p-ATF2 expression (Fig. 5B-D). Taken together, these results suggested that the JNK signaling pathway may be another 

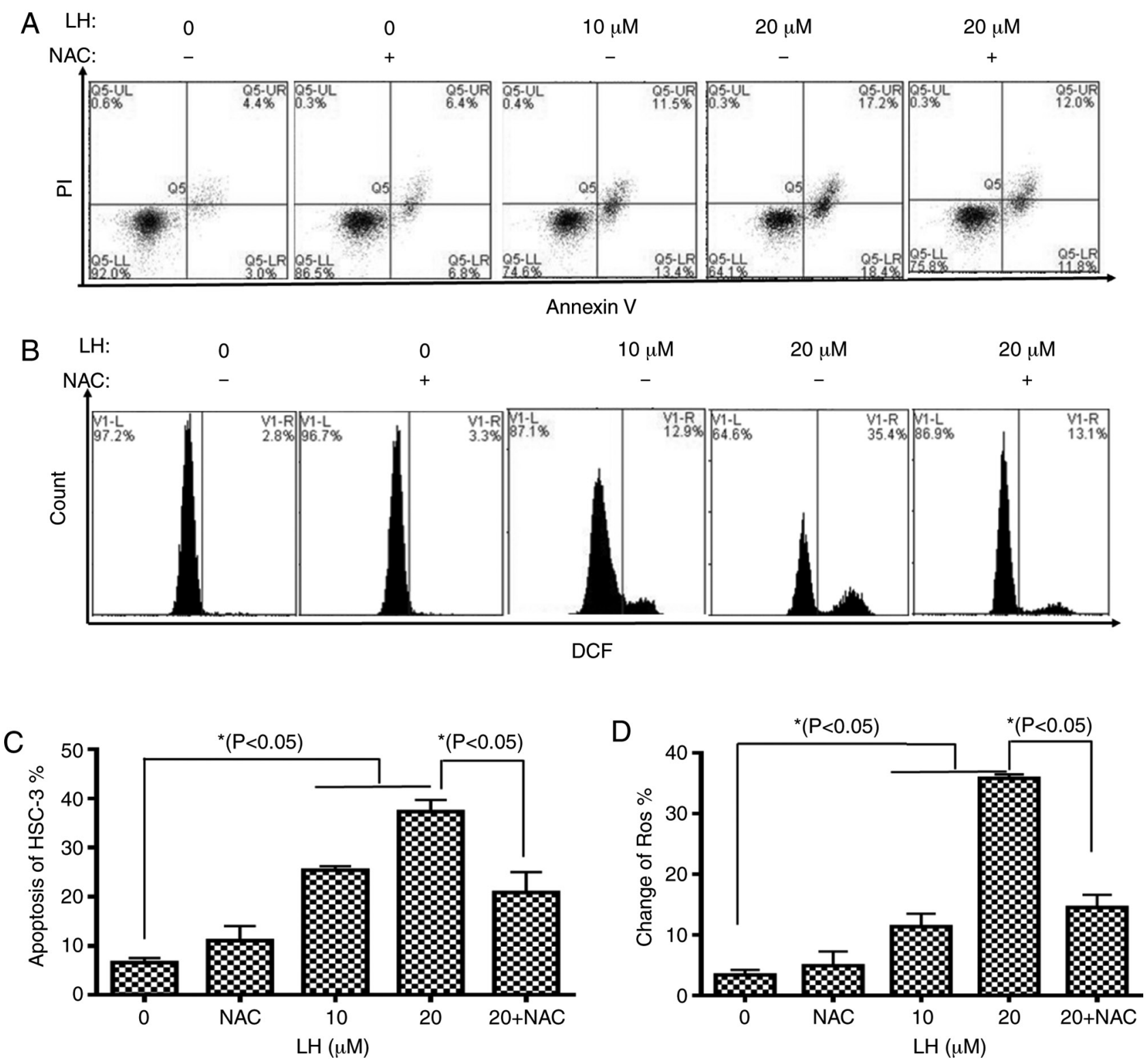

Figure 3. LH induces apoptosis mediated by ROS in HSC-3 cells. (A) Apoptotic effects of LH on HSC-3 cells were determined via dual staining with

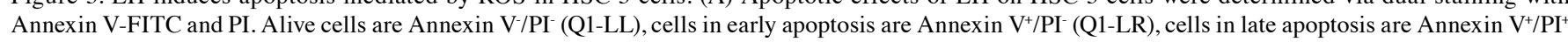
(Q1-UR) and necrotic cells are Annexin V//PI' (Q1-UL). (B) Intracellular ROS levels were investigated using the ROS-detecting fluorescence dye, DCFH-DA and flow cytometric analysis. (C) Population of apoptotic cells (Q1-LR and Q1-UR) was evaluated using histogram analyses. (D) Changes in ROS were investigated via histogram analyses. " $\mathrm{P}<0.05$ was considered to indicate a statistically significant difference using Dunnett's test for multiple group comparisons with the control group $(0 \mu \mathrm{M} \mathrm{LH})$ and Tukey's test for comparisons between group differences (groups, 20 and $20+\mathrm{NAC}$ ). LH, Lycorine hydrochloride; ROS, reactive oxygen species.

pathway involved in LH-induced HSC-3 cell apoptosis mediated by ROS.

\section{Discussion}

OSCC is a malignant tumor type that remains a major threat to human health and is associated with a high morbidity and a poor 5-year survival rate. Chemotherapy is one of the methods used to assist the multimodality treatment of advanced stage OSCC. However, traditional chemotherapy rarely achieves a significant response in prolonging survival. Therefore, it is important to identify effective candidate compounds for treating OSCC. Previous studies have focused on the active ingredients of natural products from medicinal herbs that have significant pharmacological activities, including potential antitumor effects, and it was revealed that LH significantly inhibited several different tumor cells (24-26). Therefore, the present study investigated whether LH could effectively suppress the proliferation of OSCC cells. In the present study, LH inhibited the proliferation of HSC-3 cells in a time- and dose-dependent manner. In order to identify the mechanism through which LH inhibits HSC-3 cell proliferation, cell cycle and apoptosis assays were performed in HSC-3 cells following treatment with LH. It was identified that the cell cycle of LH-treated cells was arrested at the $\mathrm{G}_{0} / \mathrm{G}_{1}$ phase. In addition, the rate of apoptosis of LH-treated HSC-3 cells was significantly increased, 

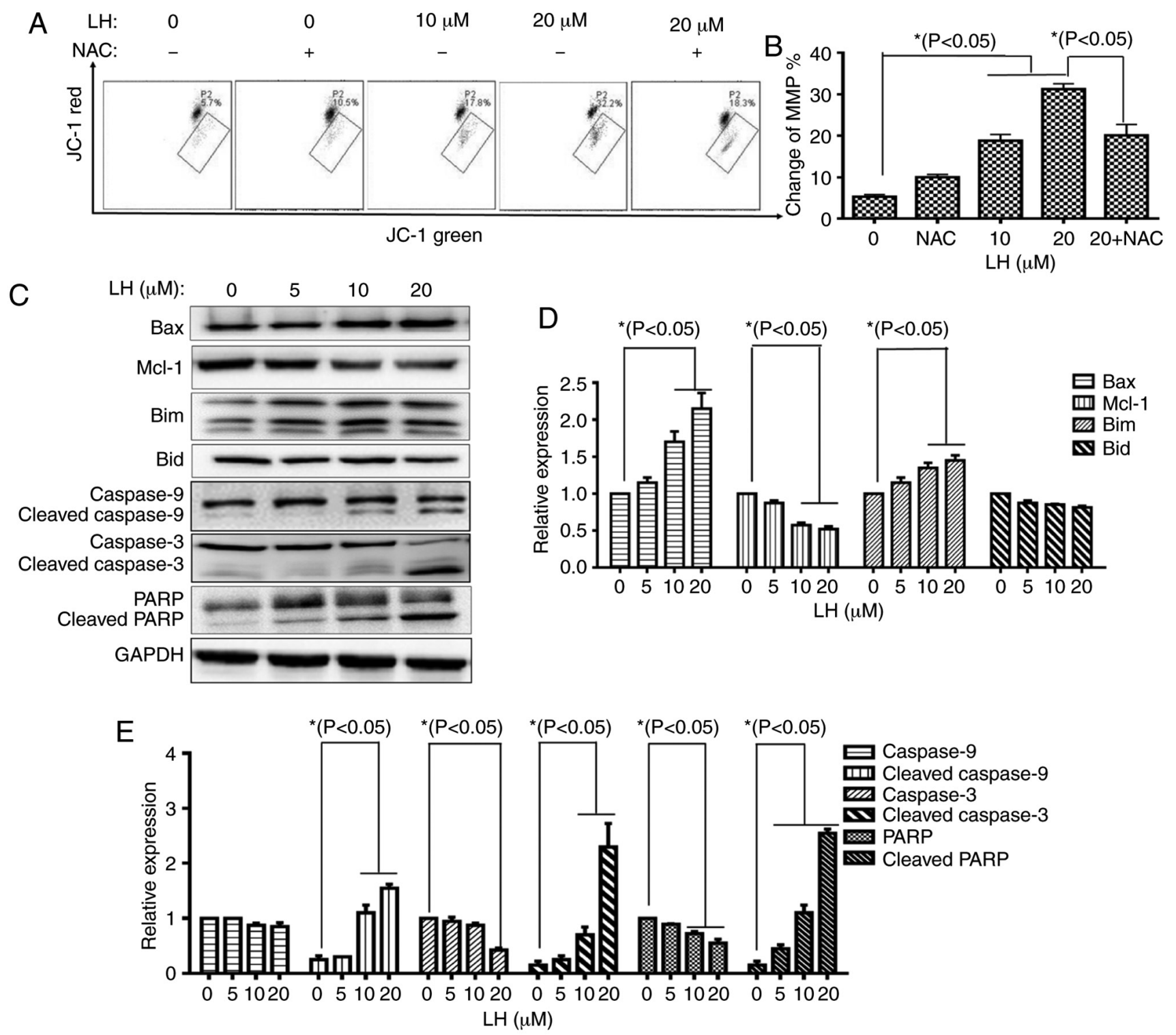

Figure 4. LH induces HSC-3 cell apoptosis via the mitochondrial pathway. (A) Changes in MMP in HSC-3 cells were detected via JC-1. Data were analyzed using Accuri C6 FCM software by measuring green $(530 \pm 30 \mathrm{~nm})$ and red $(585 \pm 40 \mathrm{~nm}) \mathrm{JC}-1$ fluorescence. MMP loss was observed by a decrease in JC-1 red fluorescence and an increase in JC-1 green fluorescence. In total, $\geq 5,000$ cells were collected and counted per sample. (B) Changes in the MMP in HSC-3 cells were investigated via histogram analyses. "P $<0.05$ was considered to indicate a statistically significant difference using Dunnett's test for multiple group comparisons with the control group $(0 \mu \mathrm{M} \mathrm{LH})$ and Tukey's test for comparisons between group differences (groups, 20 and $20+\mathrm{NAC}$ ). (C) Expression levels of the mitochondrial pathway-related apoptotic proteins were detected via western blot analysis. (D and E) HSC-3 cells were treated with LH for $24 \mathrm{~h}$, harvested and total protein lysate was subjected to western blot analysis using antibodies against GAPDH, Bax, Bim, Mcl-1, Bid, caspase-9, caspase-3 and PARP. The apoptotic protein expression was investigated via histogram analyses. ${ }^{*} \mathrm{P}<0.05$ was considered to indicate a statistically significant difference using Dunnett's test for multiple group comparisons with the control group $(0 \mu \mathrm{M} \mathrm{LH})$. LH, lycorine hydrochloride; PARP, poly(ADP-ribose) polymerase 1; Mcl-1, MCL1 apoptosis regulator, BCL-2 family member; MMP, mitochondrial membrane potential.

compared with the control group. All these data indicated that LH inhibited HSC-3 cell proliferation by inducing cellular apoptosis.

Previous studies have reported that excessive ROS may trigger mitochondrial dysfunction and cause cellular apoptosis $(24,27)$. When cells are stimulated by internal or external stress signals, the members of the Bcl-2 superfamily are affected, resulting in the upregulated expression of pro-apoptotic proteins (e.g. Bax and Bim) and downregulated expression of anti-apoptotic proteins (e.g. Mcl-1). Subsequently, the disorder of the MMP results in the opening of mitochondrial membrane pores, which causes the release of a large amounts of cytochrome $c$ from mitochondrion into the cytoplasm, forming a complex with Apaf-1 and further activating caspase- 9 , which can, in turn, activate caspase- 3 and PARP, ultimately causing apoptosis (26). The present study demonstrated that LH caused an increase in ROS production in HSC-3 cells. LH also triggered MMP disorder, as well as an increase in the protein expression levels of Bax, Bim, cleaved caspase- 9 , cleaved caspase- 3 and cleaved PARP, and a decrease in Mcl-1 expression in HSC-3 cells. Taken together, these results suggested that LH may induce HSC-3 cell apoptosis via the ROS-mediated mitochondrial apoptotic pathway. 
A

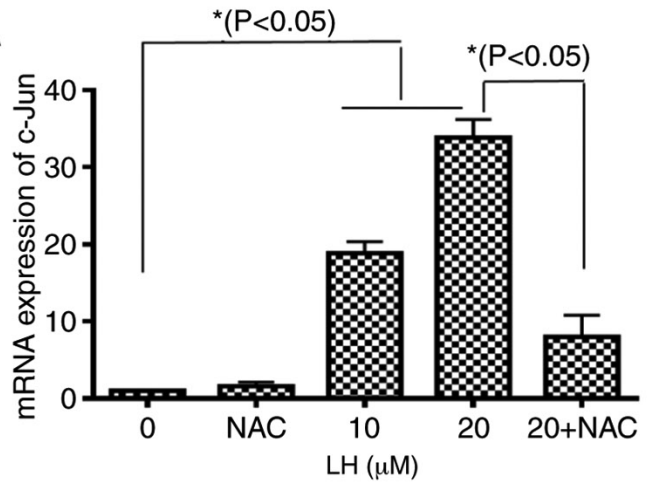

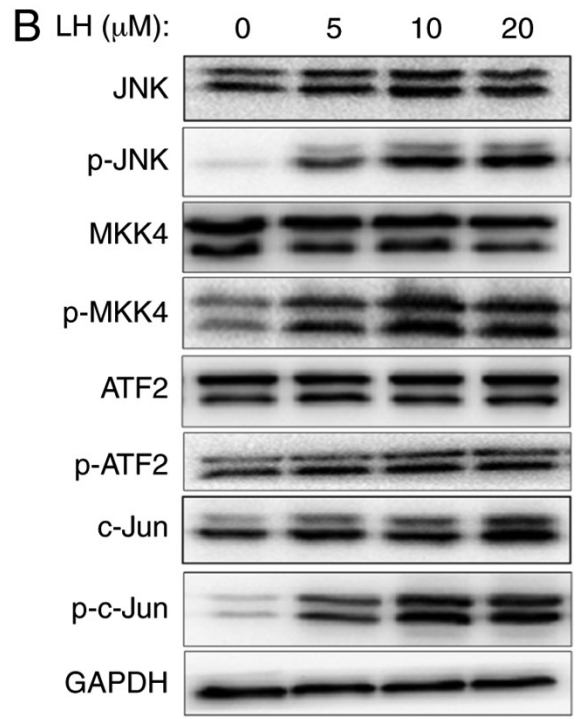
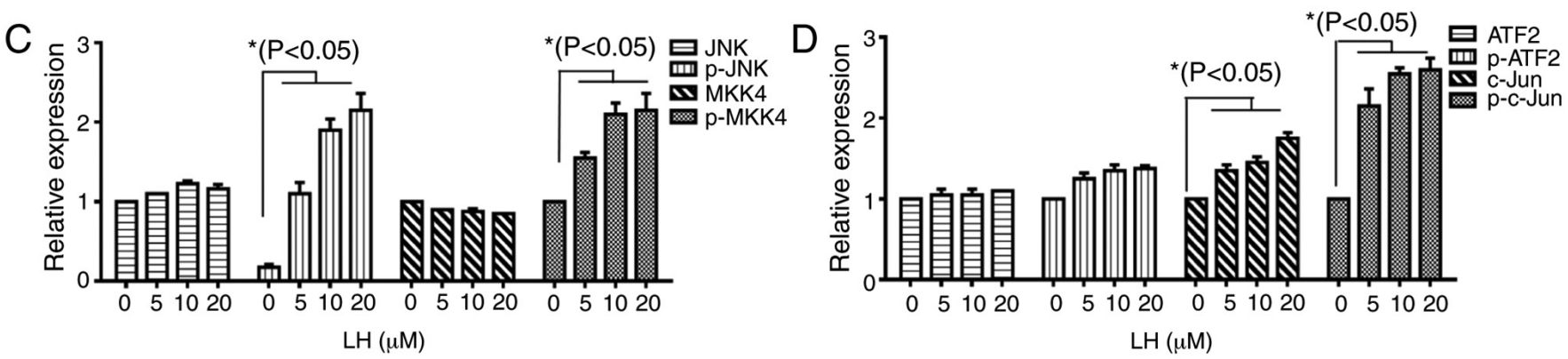

Figure 5. JNK signaling pathway is involved in LH-induced apoptosis in HSC-3 cells. (A) mRNA expression of c-Jun was detected via reverse transcription-quantitative polymerase chain reaction analysis. ${ }^{*} \mathrm{P}<0.05$ was considered to indicate a statistically significant difference using Dunnett's test for multiple group comparisons with the control group $(0 \mu \mathrm{M} \mathrm{LH})$ and Tukey's test for comparisons between group differences (groups, 20 and $20+\mathrm{NAC})$. (B) Expression levels of the JNK signaling pathway-related apoptotic proteins were detected via western blot analysis. HSC-3 cells were treated with LH for 24 h, harvested and total protein lysate was subjected to western blot analysis using antibodies against MKK4, JNK, c-Jun, ATF2 and their phosphorylation products. (C and D) Apoptotic protein expression was evaluated via histogram analyses. ${ }^{*} \mathrm{P}<0.05$ was considered to indicate a statistically significant difference using Dunnett's test for multiple group comparisons with the control group $(0 \mu \mathrm{M} \mathrm{LH})$. LH, lycorine hydrochloride; p-, phosphorylation levels.

The JNK signaling pathway has been demonstrated to serve a specific role in mediating apoptosis in several types of cancer cells (22). ROS, as an upstream regulator of JNK, serves important roles in cell proliferation, differentiation, necrosis and apoptosis, as well as other stress and inflammatory responses. Furthermore, ROS overproduction triggers mitochondrial pathway-induced cell apoptosis by activating the JNK signaling pathway and simultaneously activating proteins, including Bax, leading to damaged mitochondrial dynamics, ultimately affecting mitochondrial function and causing cell death. During this process, the phosphorylation of JNK may lead to the activation of nuclear transcription factors, including c-fos, c-Jun and ATF-2. Furthermore, the activation of JNK may regulate the Bcl-2 superfamily in apoptosis, including causing the phosphorylation of Bcl-2, resulting in Bcl-2 degradation and an inhibition of its anti-apoptotic properties. Activated JNK may also lead to changes in the MMP, resulting in a downstream cascade to induce apoptosis. MKK4, JNK and c-Jun are important members of the JNK signaling pathway. The present results suggested that the protein expression levels of p-JNK, p-MKK4 and p-c-Jun were significantly increased in LH-treated cells, accompanied by an increase of ROS. To investigate the effect of ROS on the JNK pathway, NAC, a potent antioxidant, was employed to detect the changes resulting from LH-induced pathway activation in HSC-3 cells. The present results demonstrated that NAC reversed the upregulation of the mRNA expression of c-Jun, one of the most important downstream transcription factors of the JNK signaling pathway, as well as reversing the enhanced ROS production, the disorder of MMP and HSC-3 cell apoptosis induced by $\mathrm{LH}$. Taken together, these results indicated that LH induced HSC-3 cell apoptosis via the ROS dependent activation of the JNK signaling pathway.

Based on the present data, a model for the mechanism of apoptosis induced by LH was proposed and is presented in Fig. 6. In HSC-3 cells, LH initially triggered oxidative stress, enhanced intracellular ROS, depolarized the MMP, increased the expression levels of the pro-apoptotic factors Bax/Bim, inhibited the expression level of the anti-apoptotic factor, $\mathrm{Mcl}-1$, and activated the caspases cascade of caspase-9, capsase-3 and PARP, resulting in the apoptosis of HSC-3 cells via a mitochondrial pathway. In addition, the JNK signaling pathway was involved in the apoptosis of HSC-3 cells. The increased intracellular ROS induced by LH successively stimulated the phosphorylation of MKK4 


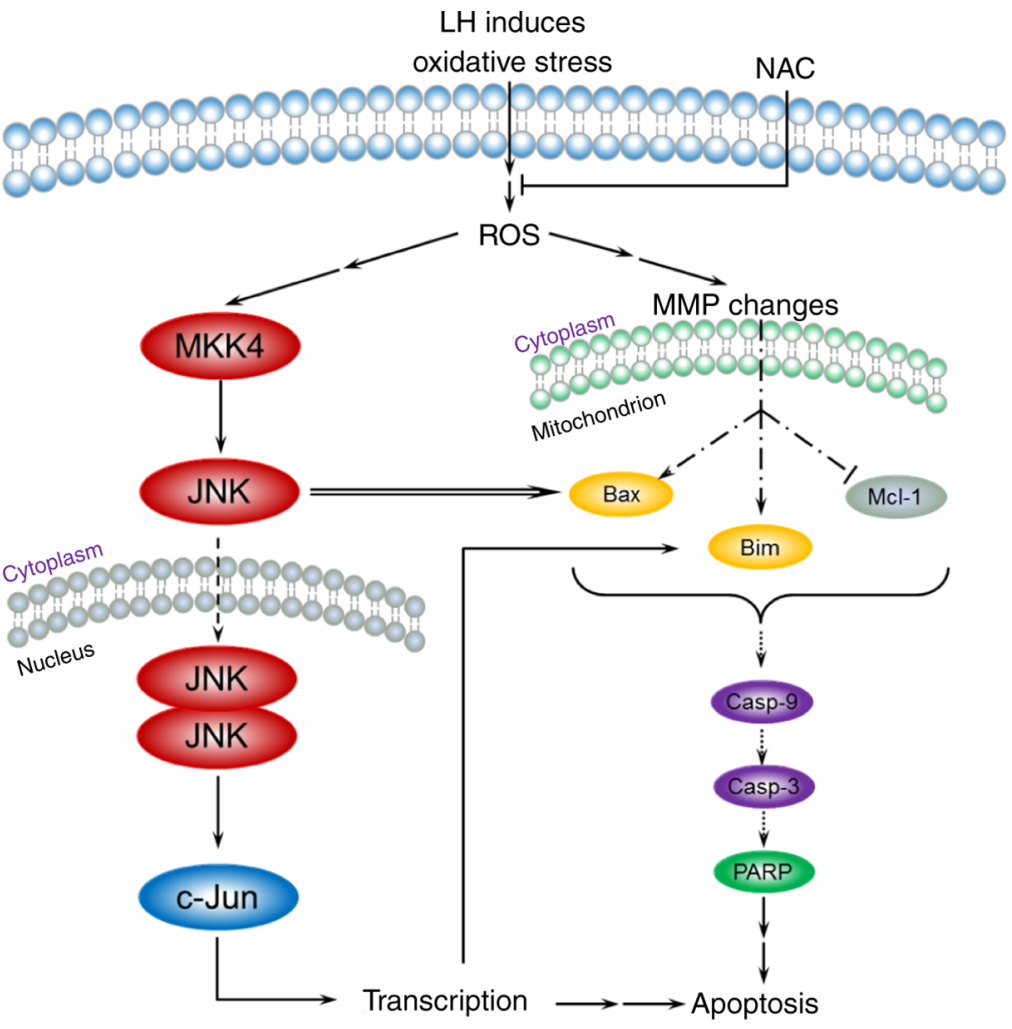

SAPK/JNK signaling pathway $\quad$ Mitochondrial pathway
Pathway diagram keys

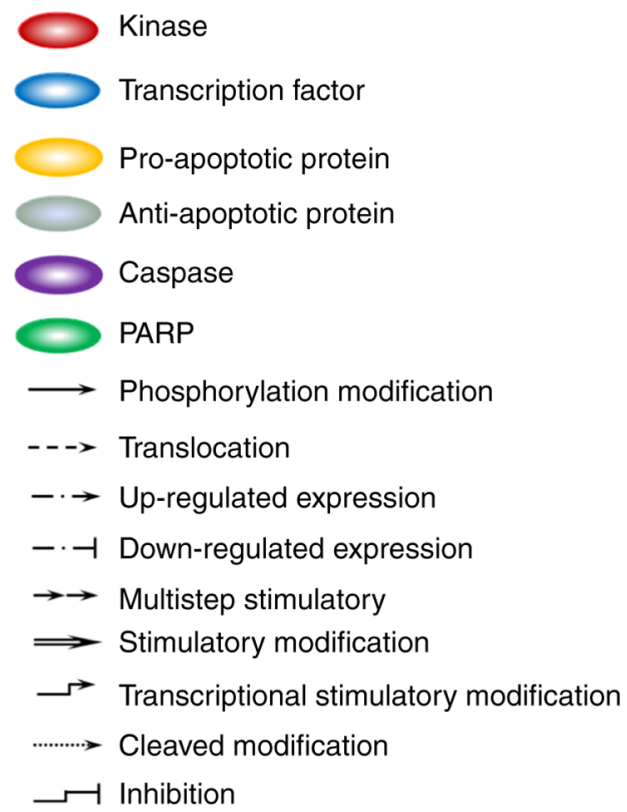

Figure 6. A model for the ROS-mediated apoptosis of LH in HSC-3 cells. The mitochondrial pathway and JNK signaling pathway are involved in the regulation of apoptosis induced by LH. LH induces oxidative stress, enhances intracellular ROS, depolarizes the MMP, promotes expression of Bax/Bim, inhibits expression of Mcl-1 and activates the caspases cascade of caspase-9, caspase-3 and PARP, resulting in apoptosis of HSC-3 cells via the mitochondrial pathway. Simultaneously, LH successively promotes MKK4, JNK and c-Jun phosphorylation, resulting in apoptosis via the JNK signaling pathway. Furthermore, these two pathways may be connected via the interactions indicated in this model between JNK and Bax, c-Jun and Bim. ROS, reactive oxygen species; LH, lycorine hydrochloride; MMP, MMP, mitochondrial membrane potential; PARP, poly(ADP-ribose) polymerase 1.

and JNK in cytoplasm, followed by the translocation of JNK into the nucleus, which further modified the transcription factor, c-Jun, resulting in a series of transcriptional stimulatory modifications targeting apoptosis. Of note, previous studies have reported that activated c-Jun may regulate Bim transcription in the nucleus, and that the activated JNK has a distinctive feature that induces the separation of the 14-3-3 subunit from the Bax/14-3-3 protein complex in the cytoplasm $(28,29)$. Therefore, these results suggest the existence of crosstalk between the mitochondrial pathway and the JNK signaling pathway, ultimately forming an orchestrated signaling network in HSC-3 cells.

However, there are several limitations to the present study that should be noted. To begin with, the potential therapeutic target of LH remains unknown. Additionally, the anti-OSCC effects of LH were investigated only in the OSCC HSC-3 cell line, and the antitumor effects of $\mathrm{LH}$ in other OSCC cell lines and primary OSCC cells were not determined. Finally, the effect of LH in an OSCC xenograft nude mouse model has not been investigated. Further studies are currently being conducted using additional OSCC cell lines and primary cells, as well as performing experiments in a nude mouse model, to validate the effects of $\mathrm{LH}$.

In conclusion, the present study demonstrated the inhibitory effect of LH on the proliferation of OSCC HSC-3 cells.
Furthermore, the apoptosis-induced effect of LH mediated by ROS via the mitochondrial apoptotic pathway and the JNK signaling pathway could be rescued by NAC pretreatment. Therefore, these results suggested that $\mathrm{LH}$ has the potential as an anticancer agent for oxidative stress-mediated OSCC therapy, based on the results of the present cell line study.

\section{Acknowledgements}

The authors would like to thank Professor Mingbo Wu (State Key Laboratory of Biotherapy, Sichuan University) for providing human OSCC HSC-3, HSC-4, UM1 and UM2 cell lines.

\section{Funding}

The present study was supported by grants from the Sichuan Science and Technology Program (grant no. 2020YFS0321) and the Applied Basic Research Program (grant no. 2017JY0173) of Science and Technology Department of Sichuan Province, the Research and Innovation Fund for Postgraduates of Chengdu Medical College (grant no. YCX2020-16), the National Undergraduates Innovating Experimentation Project of China (grant nos. 201713705007, 201713705009, 201813705002 and 201913705003), the National Natural Science Foundation of China (grant no. 81872451). 


\section{Availability of data and materials}

All data generated or analyzed during this study are included in this published article.

\section{Authors' contributions}

MHL, PY and CL conceived and designed the experiments; XL, CL, TTW, YSS KY, PWJ, STS, WXZ performed the experiments; PY, MHL and KZ analyzed the data and wrote the manuscript. All authors read and approved the final manuscript.

\section{Ethics approval and consent to participate}

Not applicable.

\section{Patient consent for participation}

Not applicable.

\section{Competing interests}

The authors declare that that they have no competing interests.

\section{References}

1. Bray F, Ferlay J, Soerjomataram I, Siegel RL, Torre LA and Jemal A: Global cancer statistics 2018: GLOBOCAN estimates of incidence and mortality worldwide for 36 cancers in 185 countries. CA Cancer J Clin 68: 394-424, 2018.

2. Ferlay J, Colombet M, Soerjomataram I, Mathers C, Parkin DM, Piñeros M, Znaor A and Bray F: Estimating the global cancer incidence and mortality in 2018: GLOBOCAN sources and methods. Int J Cancer 144: 1941-1953, 2019.

3. Wei M, Wu Y, Liu H and Xie C: Genipin induces autophagy and suppresses cell growth of oral squamous cell carcinoma via PI3K/AKT/MTOR pathway. Drug Des Devel Ther 14: 395-405, 2020.

4. Su L, Wang S, Yuan T, Xie X, Fu X, Ji P, Zhong L and Liu W: Anti-oral squamous cell carcinoma effects of a potent TAZ inhibitor AR-42. J Cancer 11: 364-373, 2020.

5. Xu Z, Jiang P and He S: Identification for exploring underlying pathogenesis and therapy strategy of oral squamous cell carcinoma by bioinformatics analysis. Med Sci Monit 25: 9216-9226, 2019.

6. Bernier J, Domenge C, Ozsahin M, Matuszewska K, Lefèbvre JL, Greiner RH, Giralt J, Maingon P, Rolland F, Bolla M, et al: Postoperative irradiation with or without concomitant chemotherapy for locally advanced head and neck cancer. N Engl J Med 350: 1945-1952, 2004.

7. Bernier J, Cooper JS, Pajak TF, van Glabbeke M, Bourhis J, Forastiere A, Ozsahin EM, Jacobs JR, Jassem J, Ang KK and Lefèbvre JL: Defining risk levels in locally advanced head and neck cancers: A comparative analysis of concurrent postoperative radiation plus chemotherapy trials of the EORTC (\#22931) and RTOG (\# 9501). Head Neck 27: 843-850, 2005.

8. Ju WT, MaHL,Zhao TC,Liang SY,Zhu DW, Wang LZ,Li J,Zhang ZY, Zhou G and Zhong LP: Stathmin guides personalized therapy in oral squamous cell carcinoma. Cancer Sci 111: 1303-1313, 2020.

9. Thiagarajan S, Dhar H, Bhattacharjee A, Fatehi KS, Shah SB, Chaukar D, Nair D, Deshmukh A, Prabhash K, Joshi A, et al: Patterns of failure and outcomes in cT4 Oral squamous cell carcinoma (OSCC) undergoing upfront surgery in comparison to neo-adjuvant chemotherapy (NACT) followed by surgery: A matched pair analysis. Oral Oncol 100: 104455, 2020.

10. Utaipan T, Boonyanuphong P, Chuprajob T, Suksamrarn A and Chunglok W: A trienone analog of curcumin, 1,7-bis(3-hydroxyphenyl)-1,4,6-heptatrien-3-one, possesses ROS- and caspase-mediated apoptosis in human oral squamous cell carcinoma cells in vitro. Appl Biol Chem 63: 7, 2020
11. Wang C, Liu XQ, Hou JS, Wang JN and Huang HZ: Molecular mechanisms of chemoresistance in oral cancer. Chin J Dent Res 19: 25-33, 2016.

12. Shah O'Brien P, Xi Y, Miller JR, Brownell AL, Zeng Q, Yoo GH, Garshott DM, O'Brien MB, Galinato AE, Cai P, et al: Disulfiram (Antabuse) activates ROS-dependent ER stress and apoptosis in oral cavity squamous cell carcinoma. J Clin Med 8: 611, 2019.

13. Yu CI, Chen CY, Liu W, Chang PC, Huang CW, Han KF, Lin IP, Lin MY and Lee CH: Sandensolide induces oxidative stress-mediated apoptosis in oral cancer cells and in zebrafish xenograft model. Mar Drugs 16: 387, 2018.

14. Dias RB, de Araújo TBS, de Freitas RD, Rodrigues ACBDC, Sousa LP, Sales CBS, Valverde LF, Soares MBP, Dos Reis MG, Coletta RD, et al: $\beta$-Lapachone and its iodine derivatives cause cell cycle arrest at $\mathrm{G} 2 / \mathrm{M}$ phase and reactive oxygen species-mediated apoptosis in human oral squamous cell carcinoma cells. Free Radic Biol Med 126: 87-100, 2018.

15. Ansari SS, Sharma AK, Soni H, Ali DM, Tews B, König R, Eibl H and Berger MR: Induction of ER and mitochondrial stress by the alkylphosphocholine erufosine in oral squamous cell carcinoma cells. Cell Death Dis 9: 296, 2018.

16. Cao Z, Yu D, Fu S, Zhang G, Pan Y, Bao M, Tu J, Shang B, Guo P, Yang P and Zhou Q: Lycorine hydrochloride selectively inhibits human ovarian cancer cell proliferation and tumor neovascularization with very low toxicity. Toxicol Lett 218: 174-185, 2013.

17. Ji Y, Yu M, Qi Z, Cui D, Xin G, Wang B, Jia W and Chang L: Study on apoptosis effect of human breast cancer cell MCF-7 induced by lycorine hydrochloride via death receptor pathway. Saudi Pharm J 25: 633-637, 2017

18. Xin G, Yu M, Hu Y, Gao S, Sun Y, Yu W, He J and Ji Y: Effect of lycorine on the structure and function of hepatoma cell membrane in vitro and in vivo. Biotech Biotechnol Equip 34: 104-114, 2020.

19. Lamoral-Theys D, Andolfi A, Van Goietsenoven G, Cimmino A, Le Calvé B, Wauthoz N, Mégalizzi V, Gras T, Bruyère C, Dubois J, et al: Lycorine, the main phenanthridine Amaryllidaceae alkaloid, exhibits significant antitumor activity in cancer cells that display resistance to proapoptotic stimuli: An investigation of structure-activity relationship and mechanistic insight. J Med Chem 52: 6244-6256, 2009.

20. Wang C, Wang Q, Li X, Jin Z, Xu P, Xu N, Xu A, Xu Y, Zheng S, Zheng J, et al: Lycorine induces apoptosis of bladder cancer T24 cells by inhibiting phospho-Akt and activating the intrinsic apoptotic cascade. Biochem Biophys Res Commun 483: 197-202, 2017.

21. Livak KJ and Schmittgen TD: Analysis of relative gene expression data using real-time quantitative PCR and the 2(-Delta Delta C(T)) method. Methods 25: 402-408, 2001.

22. Sharma A, Boise LH and Shanmugam M: Cancer metabolism and the evasion of apoptotic cell death. Cancers (Basel) 11: 1144, 2019.

23. L Z: Progress on comprehensive treatment of nasopharyngeal cancer. Cancer Res Prev Treat 46: 667-671, 2019.

24. An W, Lai H, Zhang Y, Liu M, Lin X and Cao S: Apoptotic pathway as the therapeutic target for anticancer traditional Chinese medicines. Front Pharmacol 10: 758, 2019.

25. Roy M, Liang L, Xiao X, Feng P, Ye M and Liu J: Lycorine: A prospective natural lead for anticancer drug discovery. Biomed Pharmacother 107: 615-624, 2018.

26. Zhao G, Wang Y, Yang C, Zhao L, Guo L, Li L and Wei Z: Interplay between autophagy and apoptosis in lycorine hydrochloride-induced cytotoxicity of HCT116 cells. Nat Prod Commun 14: 1934578X1986210, 2019.

27. Li MH, Yang P, Yang T, Zhang K, Liu Y, Liu J, Li LM, Luo XY, Yang SX, Zou Q and Zhang CJ: A novel water-soluble benzothiazole derivative BD926 triggers ROS-mediated B lymphoma cell apoptosis via mitochondrial and endoplasmic reticulum signaling pathways. Int J Oncol 49: 2127-2134, 2016.

28. Tomicic MT, Meise R, Aasland D, Berte N, Kitzinger R, Krämer OH, Kaina B and Christmann M: Apoptosis induced by temozolomide and nimustine in glioblastoma cells is supported by JNK/c-Jun-mediated induction of the BH3-only protein BIM. Oncotarget 6: 33755-33768, 2015.

29. Uzu M, Sato H, Shimizu A, Shibata Y, Ueno K and Hisaka A: Connexin 43 enhances Bax activation via JNK activation in sunitinib-induced apoptosis in mesothelioma cells. J Pharmacol Sci 134: 101-107, 2017.

This work is licensed under a Creative Commons Attribution-NonCommercial-NoDerivatives 4.0 International (CC BY-NC-ND 4.0) License. 